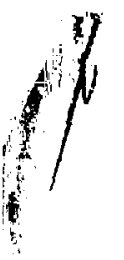

UCRL.85576

PREPRINT

\title{
MASTER
}

CONF- $810874--1$

\section{THE STUDY OF SHOCK-INDUCED SIGNALS AND COHERENT EFFECTS \\ IN SOLIDS BY MOLECULAR DYNAMICS}

\author{
ARNDLD M, KARO \\ FRANKLIIN E. HALKER \\ WARREN G. CUNNINGHAM \\ JOHN R, HARDY
}

THIS PAPER WAS PRFPAREO FOR SUBMITTAL TO

THE EIGHTH INTERNATIONAL COLLOOUIUM ON

GASDYNAMICS OF EXPLOSIONS AND REACTIVE SYSTEMS

MINSK, USSR - AUGUST 23-26, 1981

August 37,1981

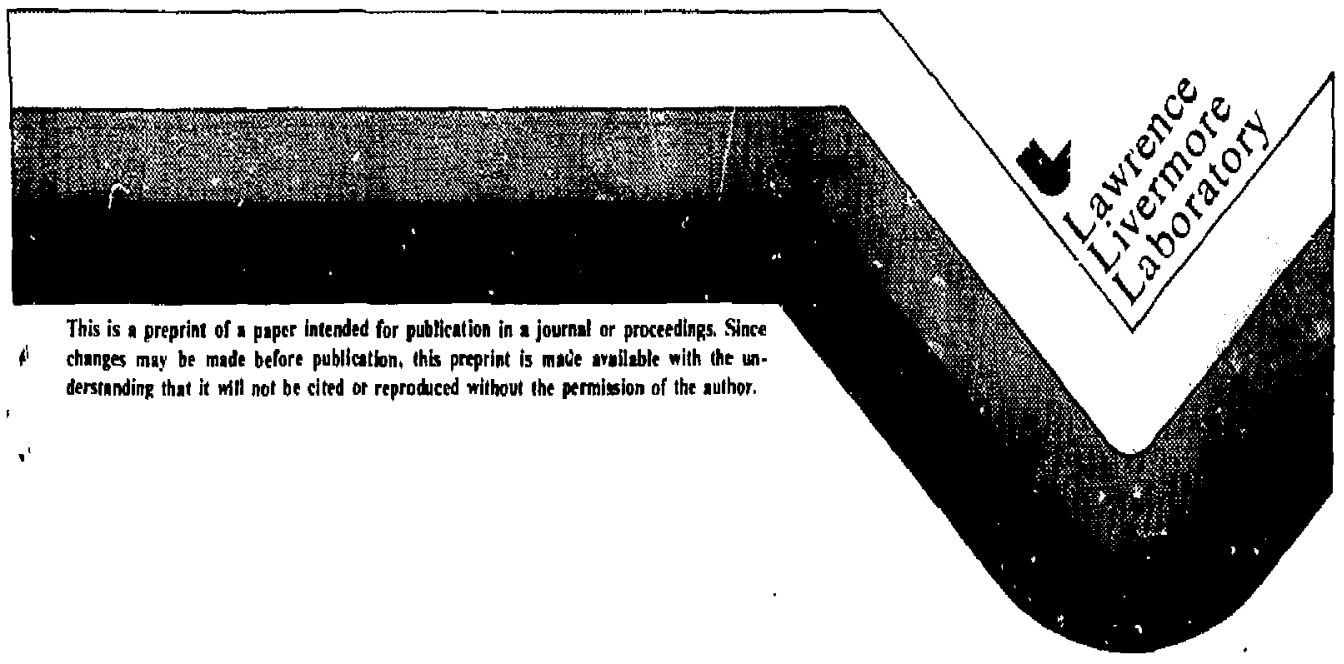


THE STUDY OF SHOCK-INDUCED SIGNALS AND COHERENT EFFECTS

IN SOLIOS BY MOLECULAR DYNAMICS*
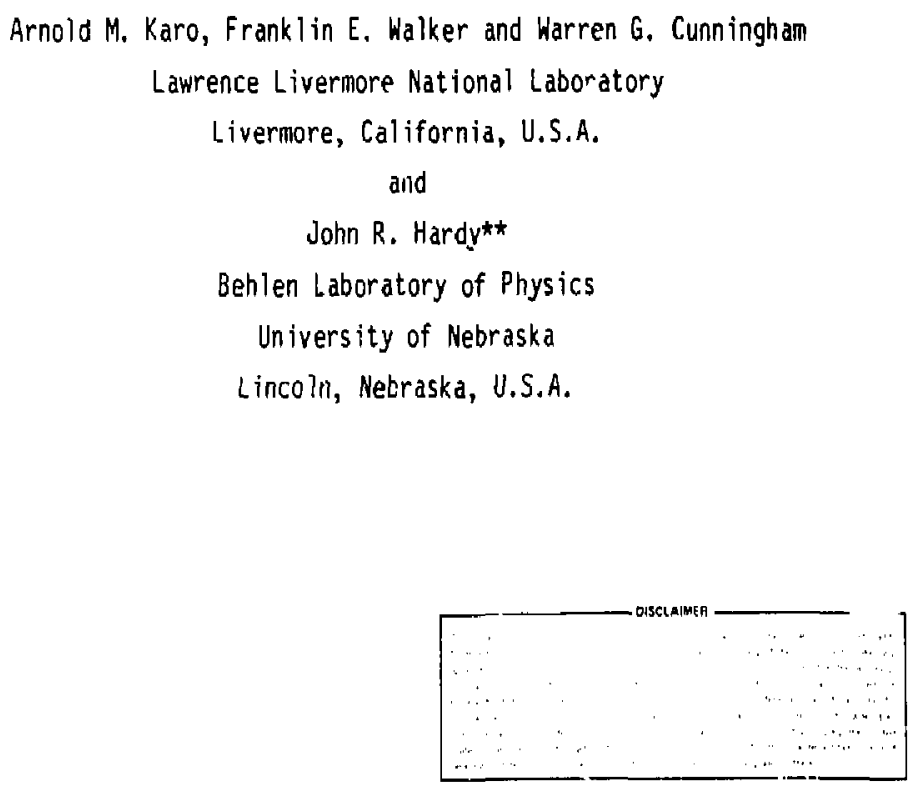

* Work performed under the auspices of the U. S. Department of Energy by the Lawrence Livermore National Laboratory under contract number W-7405-ENG-48. **Consultant for Lawrence Livermore National Laboratory. 
INTRODUCT IUN

A series of molecular dynamics calculations have been carried out that address the question of the extent to which microscopic detail can, in fact, be deduced from macroscopic measurements of shock propagation in condensed systems. The measurements we are considering are those that would typically be taken by Manganin or electromagnetic purticle-velocity gauges whose signals are then related to shock passuge through the materials being investigated, In our computer studies, we have simulated large asymmetrically shock-loaded lattices, varying both the initial temperature and the strength of shock luading.

We have found from examining the results of a wide-ranging series of such calculations that the overall pattern of everts is the consequence of a sequence of microsopic (i.e., atcmii or molecular) processes occurring in picoseconds over dimensions of angstroms. It would appear necessary, then, that experimentai techniques are required simultancously approaching such temporal and spatiai resolutions in order to deduce shock characteristics at the atomic and molecular level. Inus the attempt to meastire microscopic shock rise-times by inethods such as those referred to above is perhaps asomed to failure until gauges are developed capable of measuring displacements within the materials oi. che order of angstrins and coupled with clocks capable of measuring time to tenths of picoseconds. The effort currently expended to measure inacroscopic rise-times wouid appear to glve only a medsure of surface finish, microcrystalline structure, or void size, even with subpicosecond instrumentation. Il can also be readily seen that with shock or detonation waves propagating in solids at velocities ranging $?:$ am 5 to $9 \mathrm{~mm} / \mathrm{us}$, i.e., 5 to $9 \mathrm{~A}$ in $10^{-73} \mathrm{~s}$, and with impurities or structural irregularities no larger than $10: 2$, an apparent shock rise-time of 1 to 2 ns wclild be the shortest time measurable. Measurements made at shorter time intervals would only be probing incoherent effects associated with the inirinsic random defect structure of the material. If sample preparation could be improved to the extent that the defects or microcrystalline reatures in the material under study could be kept to no mare than $0.1 \mu$, the measured or apparent rise-time, even with subpicosecond instrumentation, would still 
$-3-$

appear to be about 0.01 to $0.01 \mathrm{~ns}$, i.e., about 100 times the periods associated with phenomena occurring on the atonic and niclecular scale. Our calculations show that microscopic rise-times associated with the shock front itself are occurring in a few measured units of $10^{-13}$ s at all points of contast between irregular surfaces. Thus the overall rise-time in a real system problem is determined merely by tlia irregularities of the impact points at the nicro-level.

The coherence associated with shocks that have been launched into a large host lattice by smaller platos that impact different regions of the surface at different times is readily observed in molecular dynamics. Lateral transfer of shock energy is minimal, even when lattices are initially thermally nigniy excited. Thus, in our calculations striking examples have been seen of the localization of shock motion within the lattice and of the degree to which such motion remains uncoupled to the lattice phonons even when the system is thermally highy excited. We have also noted in single and multiple plate calculations a very interesting demonstration of a phenonoinenun that can be referred to as a "liemory effect," in that the subsequent nistory of the shocked system "remembers" the details of the initiai loading. That is, after shock transit through the lattice and the emergence of well-separated spall from the far wall, there is a relationship displayed by the ejected material with reference to the details of the initial loading.

This is consistent with all our previous calculations on perfect lattices ${ }^{\prime}$ where shock coherence and stability are evident in the propagating disturbance. A major part of the eneryy is carried off by mcroscopic spall as the shock reaches a surface, while the rest of the system proportionally picks u's very little energy.

METHOU

In a molecular dynamics simulation of the behavior of an assembly, or array, of atoms the basic technique is the solution by computer of the Newton's law equations of motion for all of the atoms.? The forces between the atoms are given by the appropriate spatial derivatives of the potential function for the whole system. Icieally this function would be the true 
many-body potential function derived from a first-principles quantum chemistry calculation: the particle motions would be trajectaries on this $3 \mathrm{~N}$ dimensional hypersurface, where $\mathrm{N}$ is the number of atoms considered,

In practice, if ine wishes to simulate a large system, as is the case in the present studies, drastic simplification is required to make the problem tractable without requiring excessive computer time.

The simplest approximation is to represent the potentiat by a sum of short-range pairwise interactions, and it is this that we have mployed in the present studies. We have also restricted ourselves to studies on two-dimensional systems which represent further extensions of our earlier work. '

Since it is certain qualitative features of shock loading that we wish to demonstrate, these restrictions on potentials and dimensionality are unimportant. Indeed, as we shall see later, there is good reason to believe that our pair potentials provide a surprisingly good approximation to that part of the true hypersurface probed by shock loading. Moreover, by restricting ourselves to two dimensions we are able to simulate much larger crystals and thus answer questions which would require prohibitively large amounts of computer time to address for three dimensional systems. (In Ref. I we alluded to this approach as part of our general strategy.)

Given these general principles, the computational problem reduces to the solution of the $4 \mathrm{~N}$ coupled differential equatims for the particles' velocities and positions in two dimensions: the initial values of these quantities at time $t=0$ constitute the initial conditions of the probiem. SPECIFIC STUDIES

Our main objective in the present work is to examine the remarkable coherence of shock loading and its colisequences when the lattice studied is subject to localized asymmetric loading. We also wish to demonstrate that the qualitative features observed are preserved both when the shock transit distance is tripled and/or when the lattice is hot.

In order to achieve the required loading of our lattice three piates, each with a velocity of 1.4 natural units to the right ( 1 interplanar spacing per unit of $t$ ), are initially offset to the left of our lattice by three 
$-5-$

different distances. They thus strike the lattice at a sequence of times and each launches a spatially localized shock which transits the lattice and spalls material from the right-hand side. Two lattices were studied: one coritained 65 rows and 10 columns; the other 65 rows and 30 columns. Both lattices and the plates are bonded by first and second-neighbor Morse potentials which are identical for all such bonds in the systems (i.e., both lattices and plates) under study. The atomic masses are all identical and the ir value, and the bond potential, is that appropriate to the "model" lattice of Ref. 1. In order to simulate the effects of temperature, when required, the atoms are given initial random velocities at the cutset of the problem. In the case of the plates these are simply added vectorially to the initial uniform translation.

In Figs. 1-3 we show sequences of configurations from the histories of three different simulations for the thin $(65 \times 10)$ lattice.

In Fig. I both lattice and plates are cold (no initial random motion); in Fig. ? the plates are "warm" (average atomic kinetic energy 5\% of the Jond energy: this corresponds to approximately $300-400^{\circ} \mathrm{K}$ ) and the lattice is hot (average atomic kinetic energy 78\% of the bond energy); in Fig. 3 the plates are again warm, but the lattice is very hot (average atomic inetic energy $\sim 72 \%$ of the bond energy).

in all three sequences the time $t$ is shown on each configuration: the initiai configuration for all three is that shown for $t=0$ in Fig. 1 . In these and subsequent figures the atoms are shown as dots connected by 1 ines to their first neighbors in the initial configuration. As the simulations proceed these lines are retained, irrespective of separation. Thus, particu?arly at later times, widely separated pairs of atoms remain connected by these lines even though there is no significant physical bonding remaining between them.

From Fig. I we see that the successive shocks launched in the mitially quiescent thin lattice proceed through essentially independently. Each produces its own spalled fragment at the right-hand edge which carries off a major fraction of the energy imparted to the lattice by the appropriate plate impact. The bulk of the lattice remains relatively quiescent, while the 

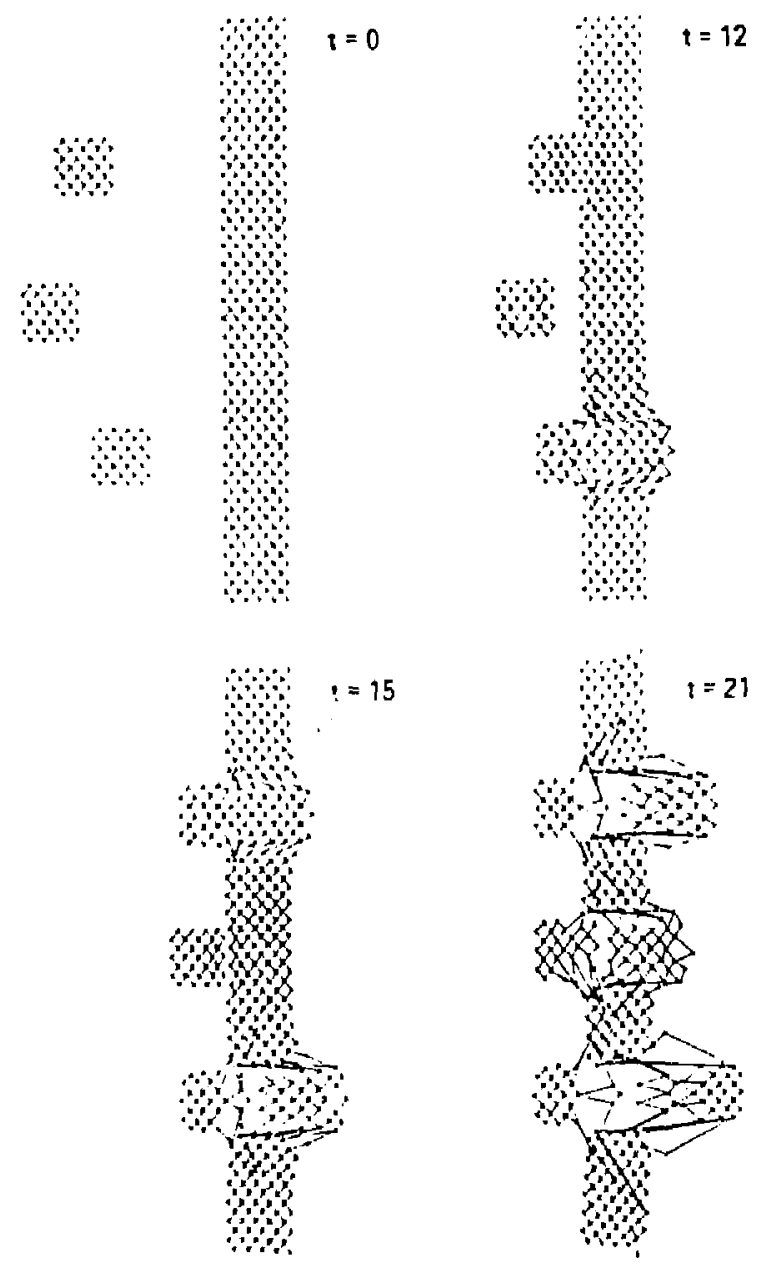

Figure 1. 

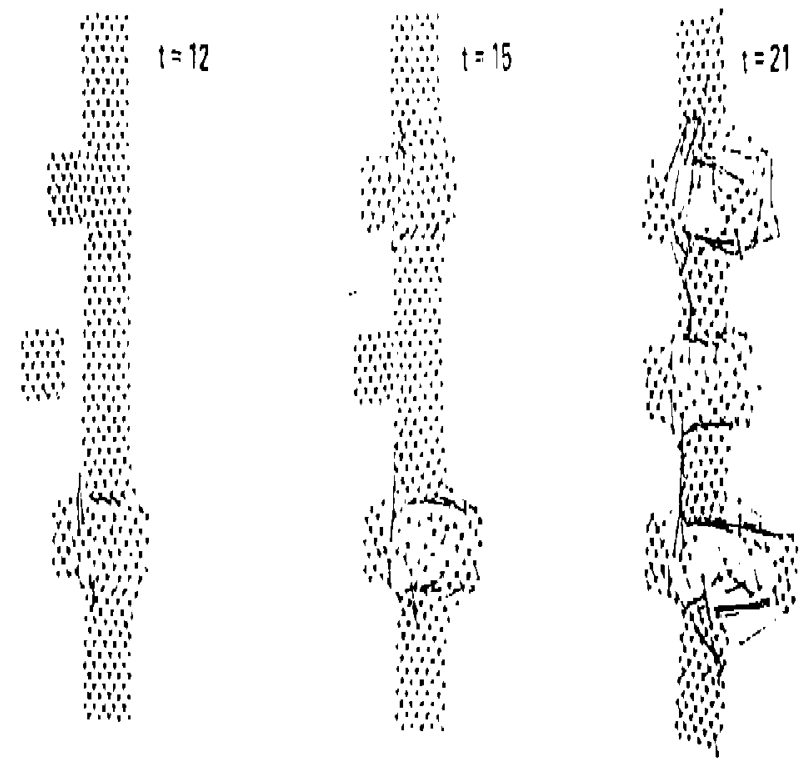

Figure?. 

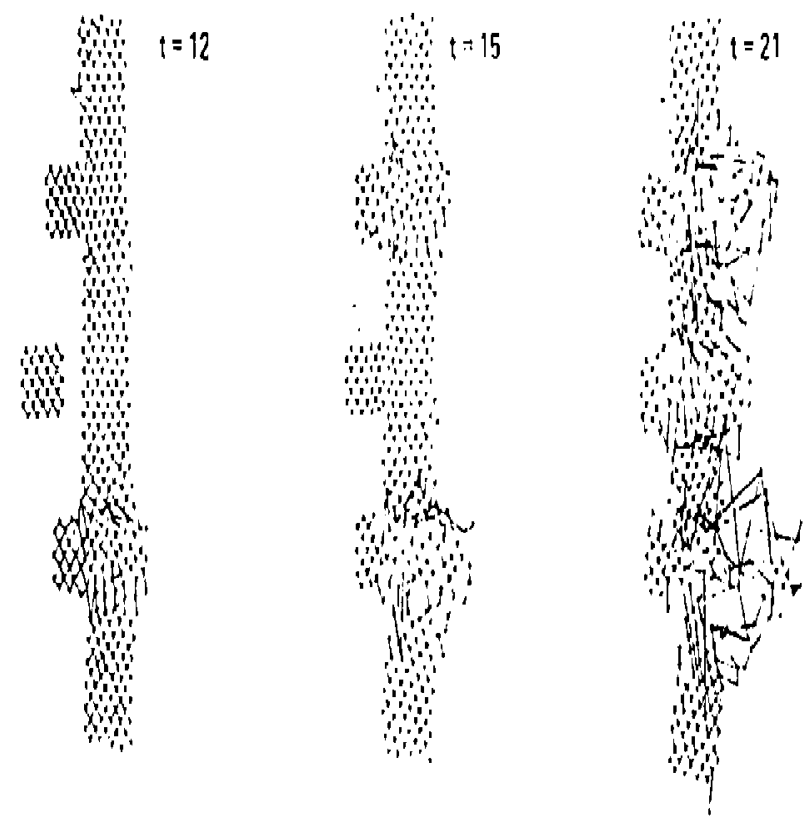

1
1

Figure 3. 
$-9-$

plate itself retains some energy as kinetic energy of recoil. The lack of transmission of energy transverse to the shock directions is most clearly evident from the remarkably symmetric nature of each spall pattern. This is best seen for the $t=21$ configuration. Here even the last (central) spall is developing essentailly as if it were taking place in isolation. From this it is clearly evident that the shock energy is highly focussed. Also, the little transverse activity that does develop appears to propagate with sonic rather than supersonic or shock speeds.

Figs. 2 and 3 show the same finite time sequences ror the hot and very not lattices. Again the clear development of successive isolated spalls is clearly apparent. There are obvious losses of symmetry, but from our earlier

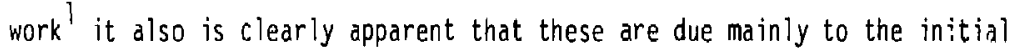
thermal motion. This persistence of spall sequence and the associated "memory effect", whereby the spall pattern "remembers" or reflects the details of the initial loading, is truly remarkable. This is particularly so for the "very hot" lattice which is in fact so tharmally excited that it is in a quas $i-1$ iquid state. In fact, if left to evolve without shock loading it spontaneous ly disintegrates. The presence of the "memory effect" in a system initially subject to such chaotic motion appears to us only to be explicable if the coupling between shock motion and thermal lattice motion is inherently a low-order process.

In order to test this belief further we carried out a series of simulations involving double rather than triple plate impact. However, the initial configuration was such that the vertical separation of the two plates was reduced by $\sim 30 \%$ in order that the traverse disturbance, produced by the firsi impact would have ample time io reach and interact with the shock produced by the second impact. The histories of three such simulaiions are shown in Figs. 4, 5 and 6 . Apart from the difference in laading these correspond exactly to the triple impact histories shown in Figs, 1, 2 and 3 respectively. These figures show clearly that the same general conclusions we reached for the triple-shocked system remain valid. For the initially quiescent system, the transverse coupl ing between the shocks is again weak: some loss of symmetry of the second shock is apparent, but its basic integrity 


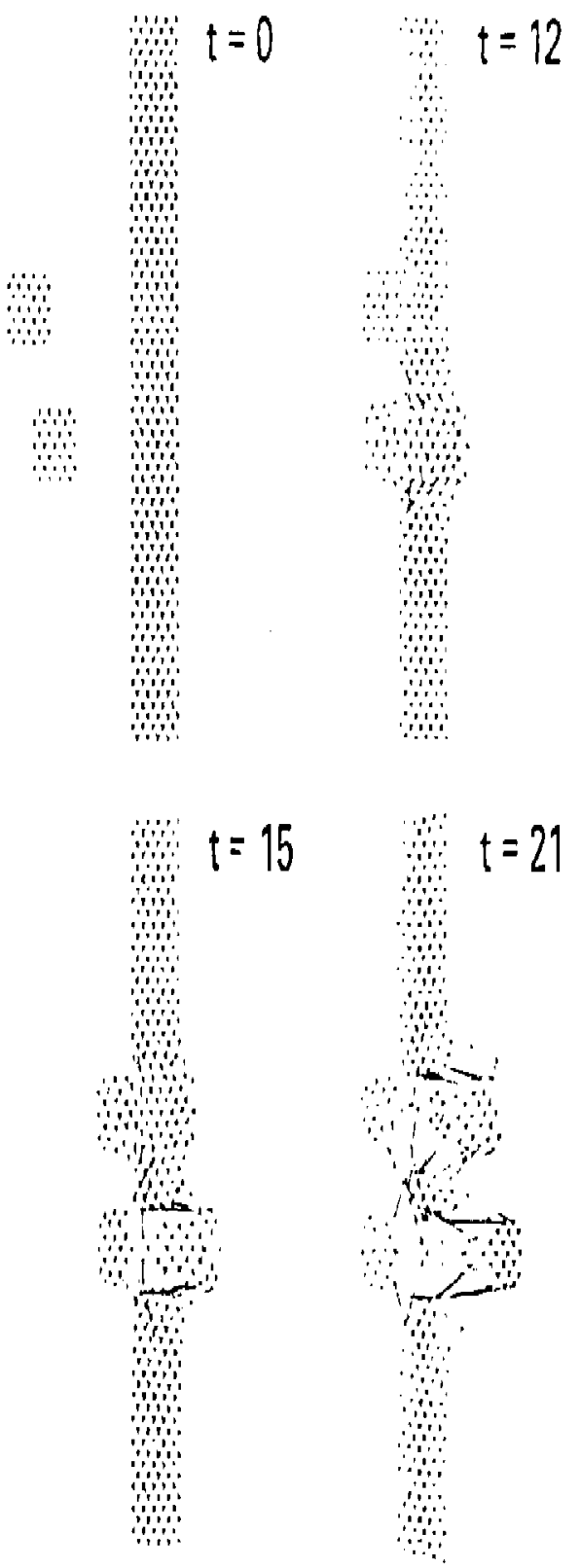

Figure 4. 

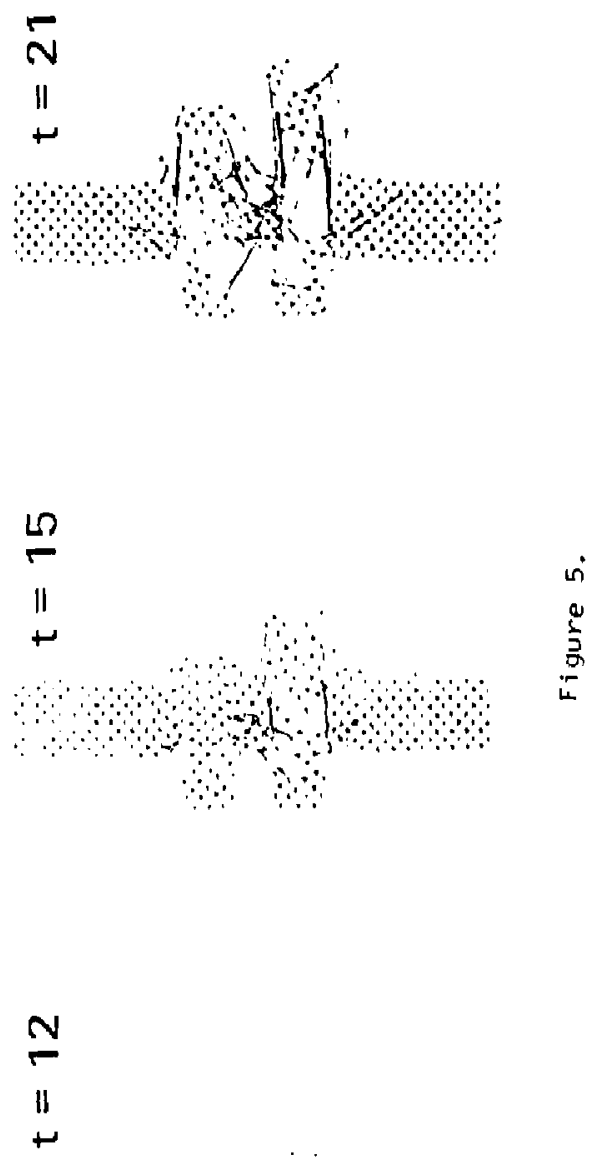

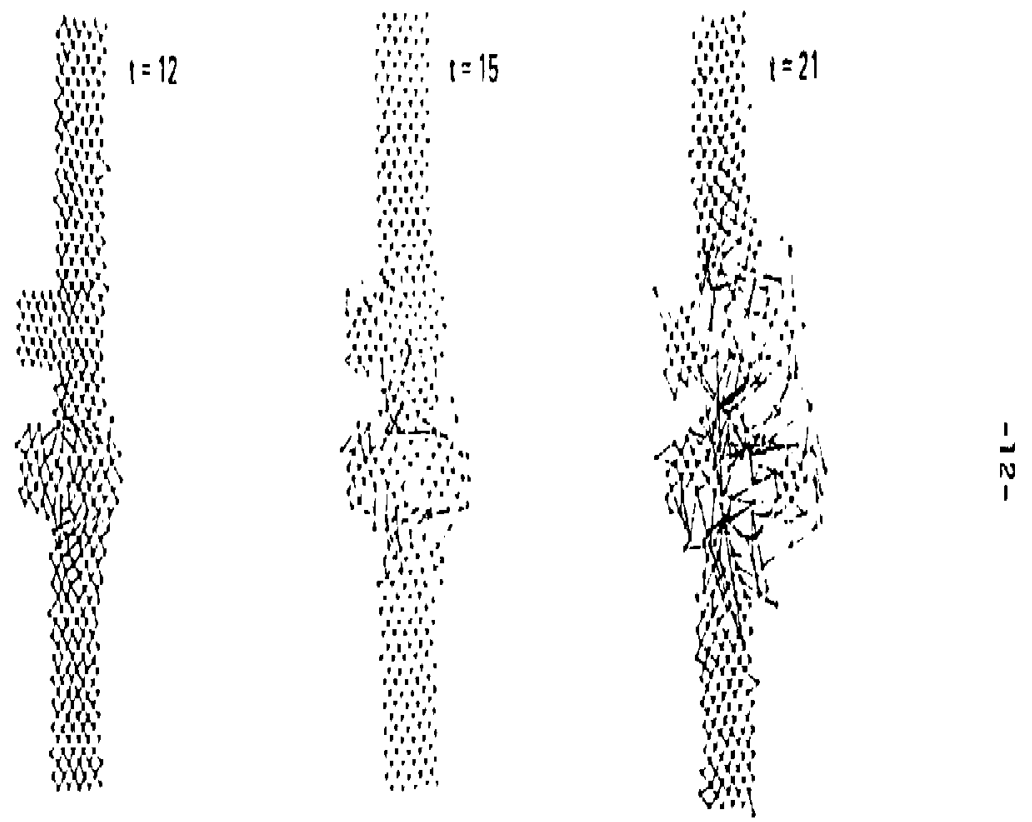

Figure 6. 
$-13-$

is unimpaired. In the cases of the "hot" systems the shack integrities are maint.dined to the same degree as they are for the corresponding triple-shocked lattices. This is entirely consistent with the results for the "cold" system: if tranverse coupling is weat for this quies int system one does not expect it to be enhanced by the presence of thermal motion.

In Figs. 7 and 8 we show the history of a triple impact on the $65 \times 30$ lattice: apart from the large size of the lattice everything else is as it was for the first simuiation for the $65 \times 10$ lattice. Thus, in the history of this initially quiescent system we see: at $t=15$ single spall is commencing due to the first (lowest) piate impact; at $t=27$ clear double spall, due to upper and lower plate impacts, is clearly developed, and the third spall is commencing from the center; at $t=53$ it is clear that we have a fina? state with clear triple spall and memory effect. For this thicker iattice mre transverse energy transfer is indicated by the clear loss of symmetry. However, it shoulu be borne in aind that any loss of zymmetry is automatically rianified as the simulation proceeds. Morenver, the horizorital crack that is apparent at $t=53$ is obvious'y a highly coherent eftect: at $t=27$ orie can see it initiating at the upper horizontal edge of the central shocked region. It is mechanical cracking and is certainly not produced by shoci energy degraded into random thermal motion. Moreover, if the upper and lower oges of the plate had been constrained, this crack might well not have developed.

In Fig. 8b the sanie time sequence of configurations is shown for a simulation identical in every way except that both plates and lattice were given the same initial "temperatures" as the plates in the $65 \times 10$ simulation. Thus both are at, or a little atove, room temperature. The history is virtually identical to that for the initially quiescent system. This indicates even more strongly than the eariier simulations that the coupling between shock and random thermal motions is very weak: it even appears that the transverse leakage of energy that does occur from shocks of finite transverse width is largely uncoupled to such thermal motion, at least at modest temperatures. 


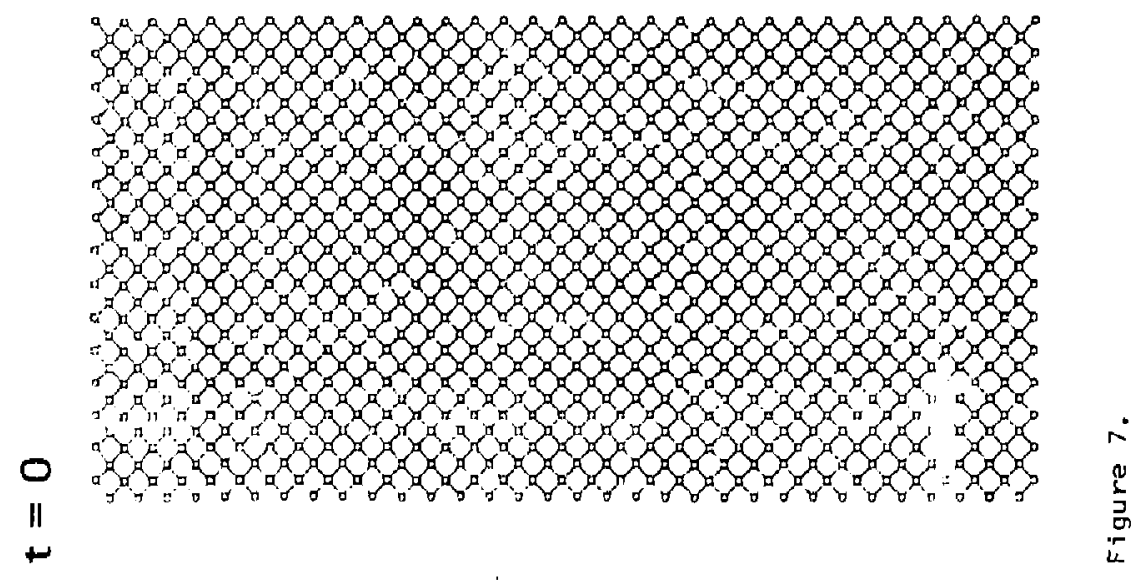

难
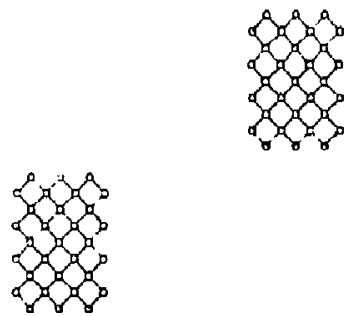
แ

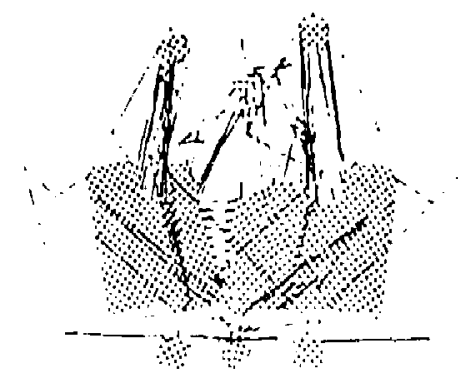

$\hat{N}$

II

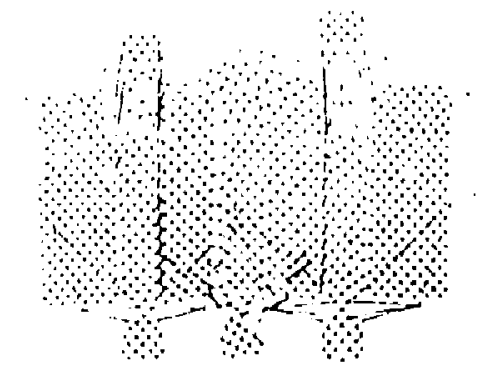

$\stackrel{2}{2}$

I

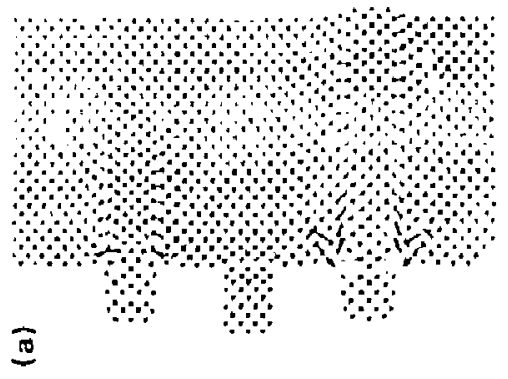

$m$
11

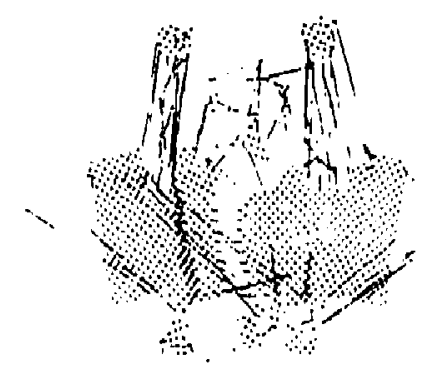

$\stackrel{N}{N}$

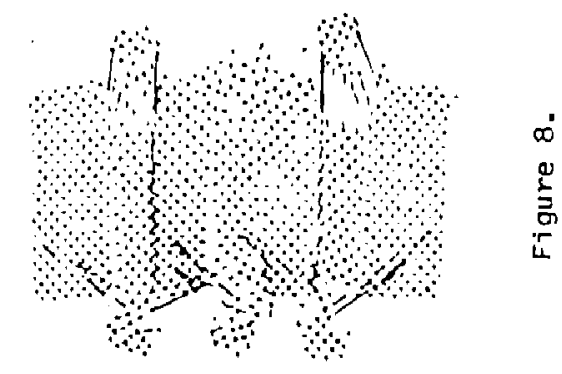

$\stackrel{10}{\sim}$

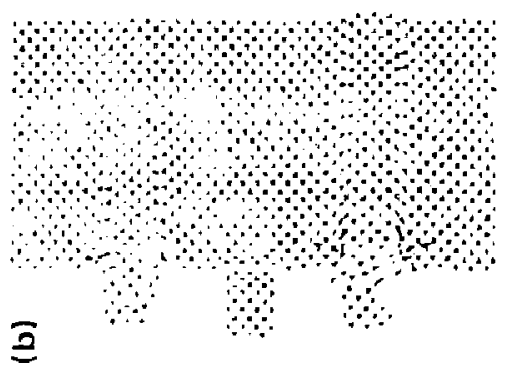


Finally, in Fig. 9, we show typical Hugoniot plots of shock versus particle velocities computed from our nolecular dynamics simulations 1 compared with experimental results. It car be seen that the two M. D. Hugoniots bracket those for a wide range of experimental materials. On this basis we can argue that the pair potential approximation, used in the present studies, provides an excellent first approximation to chat part of the true potential surface probed by shock loading. Moreover, the position of the present studies on the appropriate theoretical Hugoniot indicates that they are at the lower limits of those used in experimental studies. It thus follows that all the effects that we have described should be present in real systems under typical shock loading: they are not artifacts produced by unrealistically vioient loading in our simulations. Indeed, since these latter lie at the lower end of the Huncj jot, we would expect enhancement of the predicted effects in real systems suljected to the generally more violent loading typical of experiments. We predict this from earlier simulations 1 which have confirmed the intuitive belief that more violent loading enhance: the present type of behavior.

\section{CONCLUSIONS}

The principal result of the present studies is the demonstration of the manner in which the high degree of localization and coherence of shock energy at the atomic level affects systems multiply shocked at several locations and at several times. We find that there is remarkatly little interference between different shocks. This indicates that shcck energy is iocalized, not merely in the direction of shock propagation, but iico transverse to this direction. As a consequence of this novel risuit, indicated in earlier studies ' and confirmed by the present work, it follows that there exists a "memory effect" whereby the final spall pattern from a multiply shocked system "remembers" the details of the initial shock loading. Moreover, this memary effect persists, even when the shocked system is extremely hot.

As a consequence of these results one can infer that any macroscopic determination of rise time measures not the true microscopic rise time, which we have shown to be $\sim 10^{-12}-10^{-13} \mathrm{sec}$, but some average rise time of the overall loading which is determined by the surface irregularities of the 


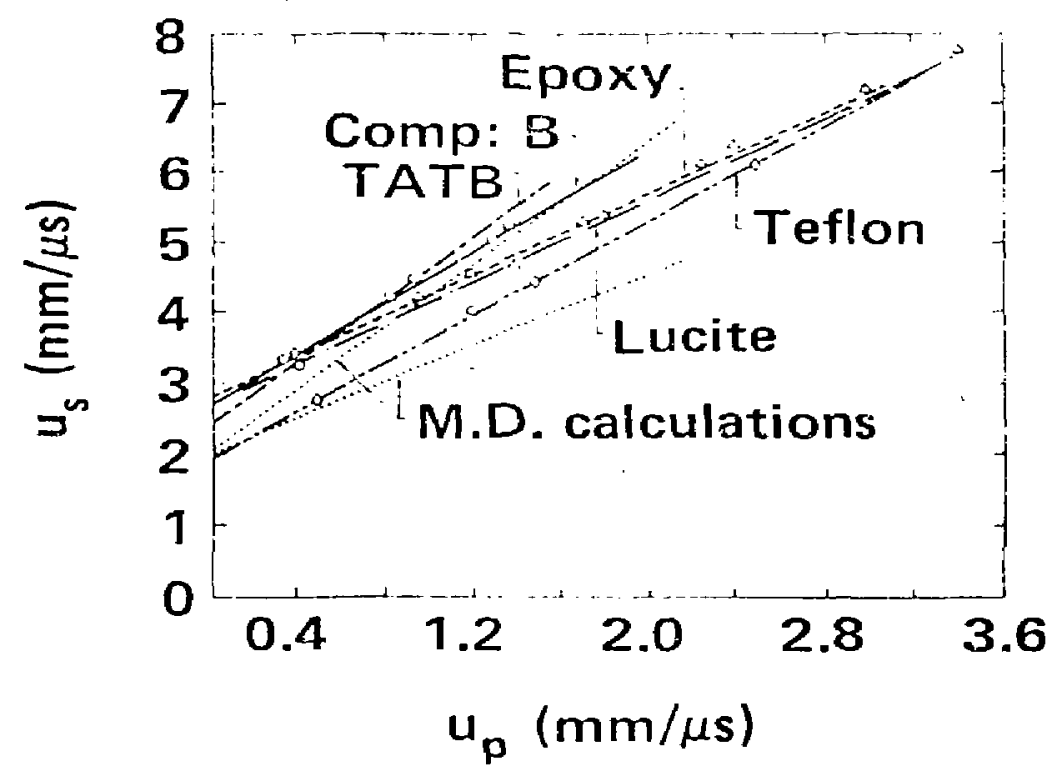

Figure 9. 
$-18-$

loading system and the shocked system. This loading history will be "remembered" by the shock as it propagates into the loaded system. Although the "memory" is not perfect, and can be degraded by irregularities in the systeri, what will be retained in the front as it propagates are the average spatial and temporal widths of the initial loading. It is these quantities that will be measured macroscopically and are erroneously regarded as "shock widths" and "rise times. The intrinsic values of these quantities are shown by our studies to be $\sim-10 \mathrm{~A}$ and $\sim 0 . i-1$ picoseconds, and these can only be probed by instrumentation having simultaneously spalial and temporal resolutions within or below these bounds.

MIS। |19ास R

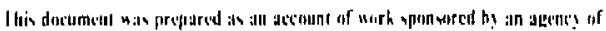

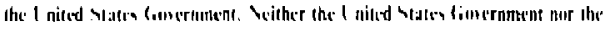

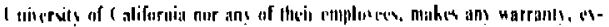

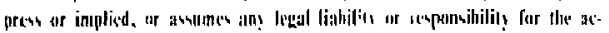

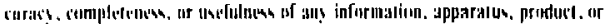

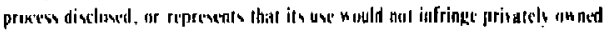

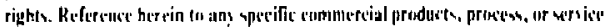

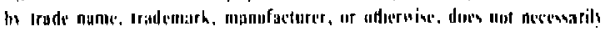

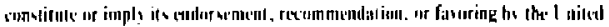

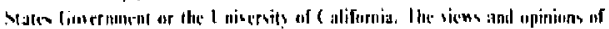

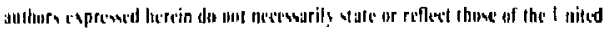

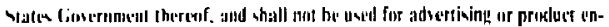
illerwintent purpous,
\end{abstract}


FIGURE CAPTIONS

Figure 1. Configurations of the initially quiescent :0 065 lattice at a sequence of times $t$ as the shocks launched by a triplet of plates, initially moving towards the lattice at $t=0$ with a velocity of 1.4 units, transit the lattice: at $t=12$ the first spall is complete, at $t=15$ the second spall is complete, and at $t=21$ the third spalled fragment is clearly separater.

Figure 2. Configurations corresponding to those in Fig. 1 except for an initial thermal motion per bond $\sim 5 \%$ of the disscciation energy for the plates and $\sim 18 \%$ of the dissuciation energy for the lattice. The spa!l sequence is strikingly similai tu that for the quiescent system.

Figure 3. Configurations corresponding to those in Fig. I except for an initial t:.ermal motion per bond $\sim 5 \%$ of the dissociation energy for the plates and $272 \%$ of the dissociation energy for the laitice.

Figure 4. Configurations of the initially quiescent $10 \times 65$ lattice at a sequence of times $t$ when it is subjected to double shock loading by two plates initially moving towards the lattice with velocities of 1.4 units at $t=0$. At $t=12$ the first spall has clearly commenced. At $t=15$ the first spall is essentially complete and the second spall is commencing. At $t=21$ the second spall is fully developed. From the $t=21$ configuration it can be seen that the second spall is very little influenced by the first shock even though the interplate spacing is only two-thirds of that used for the triple impact. 
$-20-$

Figure 5. Configurations corresponding to those in Fig. 4 except for an initial thermal motion per bond $\sim 8 \%$ of the dissociation energy for the lattice and $\sim 5 \%$ for the plates.

Figure 6. Configurations corresponding to those in Fig. 4 except for an initial thermal motion per bond $\sim 72 \%$ of the dissociation energy for the lattice and $25 \%$ for the plates.

Figure 7. Configuration of the $30 \times 65$ lattice system and associated triplet of laading plates.

Figure 8. Configurations of the Lriple-loaded $30 \times 65$ system at a sequence of times $t$ for a non-thernal and a thenind situation: (a) initially quiescent, and (b) therild) motion per bund $\sim 5 \%$ of the dissociation energy. For both systems the first spall is about to commence at $t=15$, and at $t=27$ double spall is clearly apparent. At $t=53$ we see the finai state of both systems.

Figure 9. Theuretical Hugoniots for t'vo different lattice mudels compared with experimental data. The position of our systems is indicated by the arrow pointing to the upper M. D. Hugoniot, corresponding to our lattice. 